\title{
Risk Factors for Anastomotic Leakage Following Total or Subtotal Colectomy
}

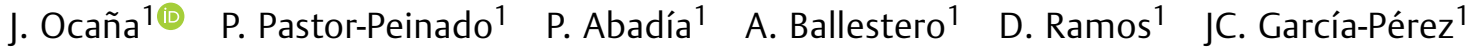 \\ JM. Fernández-Cebrián ${ }^{1} \quad$ J. Die $^{1}$ \\ ${ }^{1}$ Department of General and Digestive Surgery, Division of \\ Coloproctology, Ramón y Cajal University Hospital, Madrid, Spain. \\ Address for correspondence J. Ocaña, MD, Department of General \\ and Digestive Surgery, Ramón y Cajal University Hospital, Ctra. \\ Colmenar Viejo, Km 9.100-28034, Madrid, Spain \\ J Coloproctol 2022;42(1):38-46. \\ (e-mail: jocajim@gmail.com).
}

\begin{abstract}
Introduction A higher rate of anastomotic leakage (AL) is reported after ileosigmoid anastomosis (ISA) or ileorectal anastomosis (IRA) in total or subtotal colectomy (TSC) compared with colonic or colorectal anastomosis. The main aim of the present study was to assess potential risk factors for AL after ISA or IRA and to investigate determinants of morbidity.

Methods We identified 180 consecutive patients in a prospective referral, single center database, in which 83 of the patients underwent TSC with ISA or IRA. Data regarding the clinical characteristics, surgical treatment, and outcome were assessed to determine their association with the cumulative incidence of AL and surgical morbidity.

Results lleosigmoid anastomosis was performed in 51 of the patients (61.5\%) and IRA in 32 patients (38.6\%). The cumulative incidence of $A L$ was $15.6 \%$ (13 of 83 patients). $A$

Keywords

- total or subtotal colectomy

- ileorectal anastomosis

- ileosigmoid anastomosis

- anastomotic leakage higher $\mathrm{AL}$ rate was found in patients under 50 years-old $(p=0.038)$, in the electivelaparoscopic approach subgroup $(p=0.049)$, and patients in the inflammatory bowel disease (IBD) subgroup $(p=0.009)$. Furthermore, 14 patients $(16.9 \%)$ had morbidity classified as Clavien-Dindo $\geq$ IIIA.

Discussion A relatively high incidence of AL after TSC was observed in a relatively safe surgical procedure. Our findings suggest that the risk of AL may be higher in IBD patients. According to our results, identifying risk factors prior to surgery may improve short-term outcomes.
\end{abstract}

\section{Introduction}

Ileorectal anastomosis (IRA) and ileosigmoid anastomosis (ISA) following total or subtotal colectomy (TSC) are mainly performed in inflammatory bowel disease (IBD)-Crohn's disease, ulcerative colitis, and indeterminate colitis-familiar adenomatous polyposis or colonic polyposis syndromes, and colorectal cancer (CRC). Less frequently, TSC is performed in cases of refractory constipation and ischemic colitis. ${ }^{1-3}$
Anastomotic leakage (AL) is a significant complication, associated with increased mortality, reoperation, and derivative morbidity ${ }^{4,5}$ and is also related to poor long-term outcomes in oncological resections. ${ }^{6-8}$ Even though IRA and ISA are anatomically easy to perform-pelvic dissection is not mandatory, there is no tension at the anastomosis, and a blood supply is theoretically ensured-a higher risk of AL is reported after IRA or ISA (6.5-21\%) ${ }^{4,5,9}$ when compared with colonic or colorectal anastomosis, which has a lower AL rate, received

March 22, 2021

accepted after revision

August 6, 2021

published online

January 17, 2022
DOI https://doi.org/

$10.1055 / \mathrm{s}-0041-1740207$

ISSN 2237-9363.

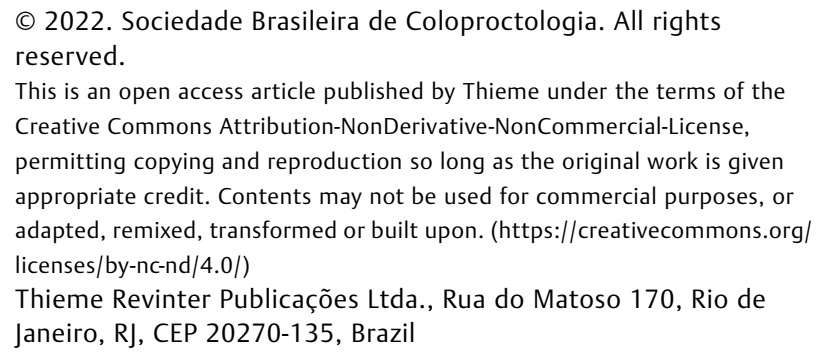

This is an open access article published by Thieme under the terms of the Creative Commons Attribution-NonDerivative-NonCommercial-License, permitting copying and reproduction so long as the original work is given appropriate credit. Contents may not be used for commercial purposes, or adapted, remixed, transformed or built upon. (https://creativecommons.org/ licenses/by-nc-nd/4.0/)

Thieme Revinter Publicações Ltda., Rua do Matoso 170, Rio de Janeiro, RJ, CEP 20270-135, Brazil 
mainly under $15 \%{ }^{4,10,11}$ Regardless of the indication, ${ }^{12}$ similar AL rates are seen after TSC in IBD (4-12\%), ${ }^{13,14}$ polyposis $(20 \%)^{15}$ and colon cancer (6-21\%).,16 Reducing AL rates might improve short and long term as well as functional outcomes after IRA or ISA. There is not enough evidence of determining factors for AL following colectomy with IRA or ISA. The main aim of the present study was to assess the potential risk factors for AL after ISA or IRA and to investigate determinants of morbidity.

\section{Methods}

\section{Patients}

All consecutive patients who underwent ISA or IRA following TSC between January 2013 and December 2019 were retrospectively identified from the prospective database of a tertiary referral center in the Ramón y Cajal University Hospital, Madrid, Spain. In case of missing data, patients' records were reviewed. We defined ISA as an anastomosis higher than promontory level, and anastomosis below this level was considered as IRA. The main indications for colectomy in eligible patients were CRC, IBD, polyposis syndromes, and refractory constipation. We defined $\mathrm{AL}$ as a defect in the integrity of the intestinal wall at the anastomotic site leading to a communication of the intra- and extraluminal, or to a pelvic abscess adjacent to the anastomosis, according to the definition set by the International Study Group of Rectal Cancer. ${ }^{17,18}$ When the AL required no active therapeutic intervention, they were classified as grade $\mathrm{A}$. When the $\mathrm{AL}$ required active therapeutic intervention (such as antibiotics and percutaneous drainage), but was manageable without relaparotomy, they were classified as grade $\mathrm{B}$. The $\mathrm{AL}$ requiring reintervention were classified as grade $C$. There was a postoperative follow-up of 90 days. Postoperative morbidity and mortality were considered within 90 days after surgery. The Clavien-Dindo classification of morbidity was used to grade postoperative complications. ${ }^{19}$ Readmission was considered within 90 days in those patients who presented surgicalassociated morbidity, excluding other diagnosis. Patients under 18-years-old, terminal ileostomy after TSC, and two-stage procedure (IRA or ISA with diverting ileostomy) were excluded. The anastomosis was performed according to the surgeon's preference. Demographic and clinical data were collected from each patient, including age, gender, physical status according to the American Society of Anesthesiologists' (ASA) classification, presence of cardiovascular disease, diabetes mellitus, chronic kidney failure, or other underlying diseases, planning of operation (emergent/elective), and preoperative total proteins. Surgical approach, operative variables, and type of anastomosis (handsewn or stapler) were also collected.

\section{Statistical Analysis}

The results are reported in accordance with the Strengthening the Reporting of Observational Studies in Epidemiology (STROBE) statement. ${ }^{20}$ Continuous variables were reported as the median and interquartile range, whereas categorical variables were reported as the number of patients and percentage. A univariate analysis was performed to assess the association between dependent variables (AL and postoperative morbidity) and independent variables (ISA or IRA, patients' and surgical data): continuous variables and discrete variables were analyzed by the U Mann-Whitney test and the $\mathrm{X}^{2}$ test, respectively. Statistical significance was indicated by a $p$-value $<0.05$ (2-tailed test). The IBM SPSS Statistics for Windows, Version 22.0 software (IBM, CorpArmonk, NY, USA) was used for the analyses.

\section{Results}

Of the 180 patients identified in the database (-Fig. 1), 92 were excluded as they did not have bowel reconstruction with ISA or IRA, and another 5 patients already had diverting stoma. The remaining 83 patients were included and assessed in the study. Demographic characteristics and preoperative data according to $\mathrm{AL}$ are shown in -Table 1. The cumulative incidence of $\mathrm{AL}$ was $15.6 \%$ (13 of 83), with 1 patient having grade $A, 1$ patient grade $B$, and 11 patients grade $C$. A higher rate of AL was found in patients under 50 years-old ( $p=0.038$ ). Surprisingly, no comorbidities were found to be correlated with AL in the univariate analysis. The main indication for surgery was colon cancer $(n=63 ; 75.9 \%)$. Colonic polyposis $(n=3 ; 3.6 \%)$, IBD $(n=15 ; 18.1 \%)$, and refractory constipation $(n=2,2.4 \%)$ were other reasons for surgery. Whereas the AL rate was significantly higher in the IBD group $(p=0.009)$, no significant AL rate differences were found in the emergent surgery group ( $25 \%$ vs $13.4 \% ; p=0.27$ ).

All surgical data is shown in -Table 2. Ileosigmoid anastomosis was performed in 51 patients (61.5\%), and IRA was used in 32 patients (38.6\%). No significant differences in AL rate $(p=0.21)$ were found between IRA and ISA. The anastomosis technique (handsewn vs. stapler, $10.3 \%$ vs. $18 \%$ ) and the type of anastomosis were also found not to be predictors of AL, with $p=0.31$ and $p=0.57$ respectively. Laparoscopic surgery was performed in 19 of the 83 patients (22.9\%). In the elective surgery subgroup, laparoscopic approach had higher AL rate than open surgery (27.8\% vs. $8.2 \%$ ), with $p=0.049$.

Surgical morbidity according to patient and surgical data is shown in - Table 3. Of the 83 patients, 38 (45.8\%) had no surgical complications within 90 days postoperatively (-Table 4). None of the assessed potential predictors of morbidity were found to be significant. Fourteen patients (16.9\%) had morbidity classified as Clavien-Dindo $\geq$ IIIA (moderate-severe). The distribution of exposures according to AL grade was assessed (-Table $\mathbf{3}$ ), and no potential predictors were found to be statistically significant. There weren't any deaths within 90 days after surgery. Only 2 patients were readmitted $(2.4 \%)$ in the follow-up.

\section{Discussion}

In the present study of risk factors for AL and morbidity following TSC with ISA or IRA, the overall AL rate was 15.6\%. No mortality event was recorded within 90 days postoperatively, which is similar to previous reports, with a mortality rate under $5 \%^{9,16}$ in recently published series. The mortality 


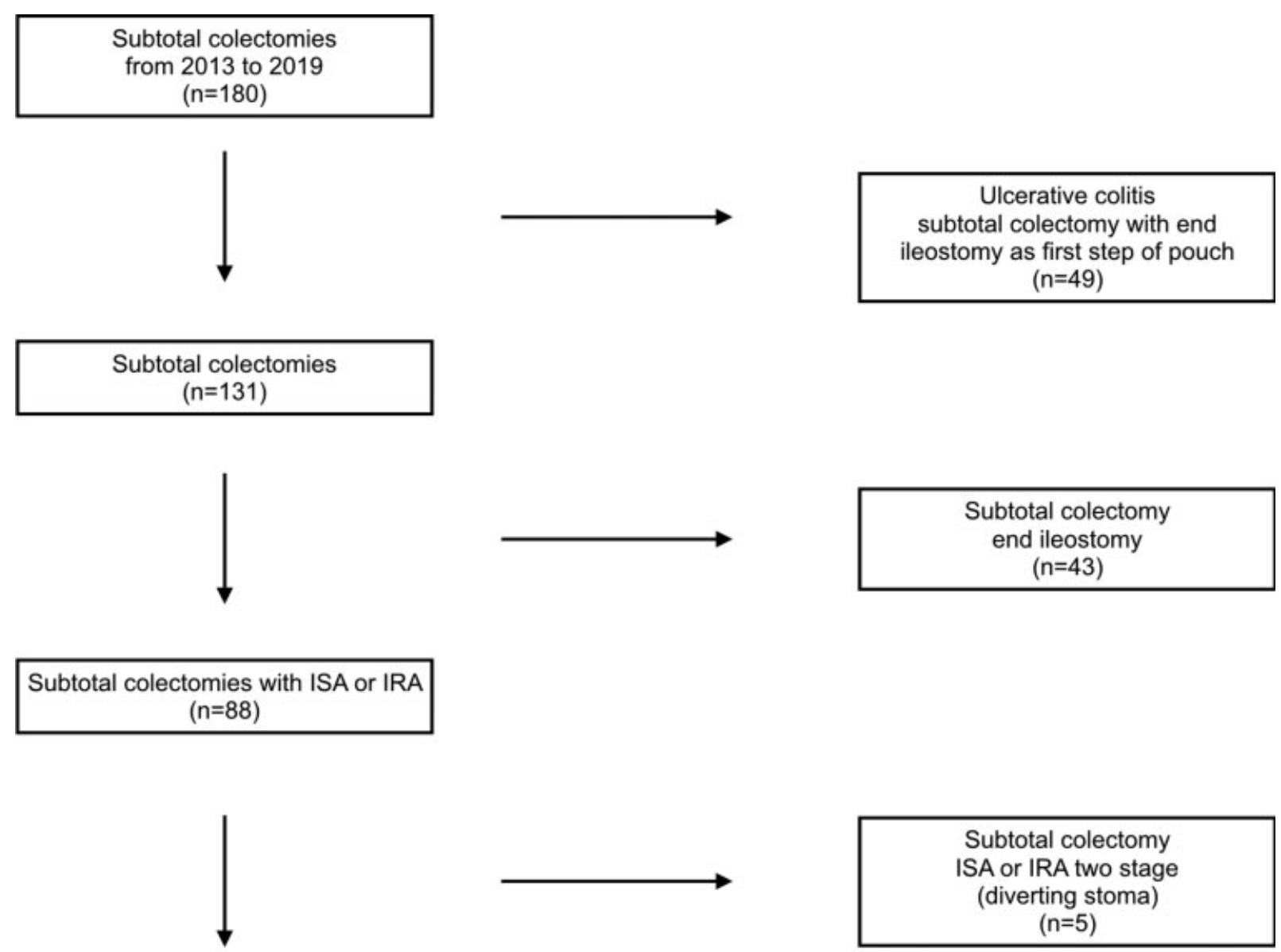

Subtotal colectomies with ISA or IRA One-stage (Final cohort) $(n=83)$

Fig. 1 Flowchart of selection.

rate might be higher in emergent surgery. ${ }^{21}$ The range of $A L$ after IRA or ISA is widely variable depending of the study design. ${ }^{5,22,23}$ The occurrence of AL in the present study was similar to the findings reported by Segelman et al., who found an AL rate of $13.2 \%$ after TSC with ISA or IRA, reporting a significantly lower AL rate (7\%) in two-stage procedures (loop ileostomy); Duclos et al. also reported a significant AL rate of $7 \%$. It might be due to a high rate of two-stage procedures (20\%), whereas in a nationwide register study, an AL rate of $13.4 \%$ was reported following TSC in colon cancer. ${ }^{4}$ Even though including two-stage procedures had improved our final results, we decided in the study design to exclude those patients to avoid potential bias in the risk factors for $\mathrm{AL}$ detection. In previous published reports of emergent TSC in obstructed left colon cancer, an incredible low AL rate was reported, under $10 \%{ }^{24-26}$ The heterogeneity of $\mathrm{AL}$ in the literature might be due to a discrepancy in the definition of AL. In our study, we adopted the definition set by the International Study Group of Rectal Cancer ${ }^{17}$ to be able to compare our results with those previously reported by others who use the same definition. ${ }^{16}$
The impact of the anastomosis (ISA or ISA) on AL is controversial. Segelman et al. ${ }^{16}$ did not find any statistical difference in one of the most recent studies (XX). In our study, there was no difference in the rate of AL between ISA and IRA, showing that the height of the anastomosis might not have a critical relevance. The difference between ISA and IRA was assessed in elective surgery, and no statistical differences were found. Even though the operation records were difficult to analyze when trying to identify which vessels were preserved, anastomosis involving the distal segment of the small bowel often has sufficient blood supply. On the other hand, in benign diseases, the superior rectal artery can be preserved, allowing sufficient blood perfusion to the anastomosis. It is still unclear if there is an impact of high ligation of the inferior mesenteric artery in the AL rate. ${ }^{27-30}$ Likewise, the impact of the perfusion of the distal part of ISA or IRA in the AL rate is also controversial.

A great number of studies have been published about risk factors for AL after colectomy, ${ }^{4,10,11,28-30}$ but the majority are focused on colorectal cancer patients. The results of IRA or ISA after TSC are mixed with other anastomosis sites, and 
Table 1 Baseline and preoperative data, and anastomosis leakage

\begin{tabular}{|c|c|c|c|}
\hline & \multicolumn{3}{|c|}{ Anastomosis leakage } \\
\hline & $\begin{array}{l}\text { Non- anastomosis } \\
\text { leakage } \mathrm{n}(\%)\end{array}$ & $\begin{array}{l}\text { Anastomosis } \\
\text { leakage } \mathrm{n}(\%)\end{array}$ & $\begin{array}{l}p \text {-value } \\
\text { OR (CI95\%) }\end{array}$ \\
\hline Age, median (range) & $66(28-82)$ & $61(39-80)$ & 0.49 \\
\hline Age: $50 y$ & & & $\begin{array}{l}0.038 \\
4.23(1.13-9.83)\end{array}$ \\
\hline Age $\geq 50 y$ & $61(88.4)$ & $8(11.6)$ & \\
\hline Age $<50 y$ & $9(64.3)$ & $5(37.5)$ & \\
\hline Gender & & & 0.48 \\
\hline Male & $50(80.6)$ & $8(13.8)$ & \\
\hline Female & $20(80)$ & $5(20)$ & \\
\hline ASA & & & 0.10 \\
\hline 1 & $23(74.2)$ & $8(25.8)$ & \\
\hline II & $37(92.5)$ & $3(7.5)$ & \\
\hline III & $9(81.8)$ & $2(18.2)$ & \\
\hline Cardiovascular disease & $13(92.2)$ & $1(7.1)$ & 0.29 \\
\hline Chronic kidney disease & $8(83.1)$ & $1(16.9)$ & 0.94 \\
\hline Diabetes mellitus & $9(90)$ & $1(10)$ & 0.58 \\
\hline Reason for surgery & & & 0,048 \\
\hline Cancer & $56(88.9)$ & $7(11.1)$ & \\
\hline Colonic polyposis & $3(100)$ & 0 & \\
\hline IBD & $9(60)$ & $6(40)$ & \\
\hline Constipation & $2(100)$ & 0 & \\
\hline Reason for surgery & & & $\begin{array}{l}0.009 \\
3.88(1.52-9.90) \\
\end{array}$ \\
\hline $\mathrm{IBD}$ & $9(60)$ & $6(40)$ & \\
\hline Non IBD & $61(89,7)$ & $7(10.3)$ & \\
\hline Planning of operation & & & 0.27 \\
\hline Elective & $58(86.6)$ & $9(13.4)$ & \\
\hline Emergent & $12(75)$ & $4(25)$ & \\
\hline $\begin{array}{l}\text { Preopearative hemoglobin }(\mathrm{mg} / \mathrm{dL} \text { ) } \\
\text { median (range) }\end{array}$ & $12.7(9.8-17.2)$ & $14(12.2-17.4)$ & 0.14 \\
\hline Preoperative total proteins $(\mathrm{g} / \mathrm{dL}$ ) & $6.05(4-7.5)$ & $6.45(4.5-8.3)$ & 0.42 \\
\hline
\end{tabular}

Abbreviations: ASA, American Society of Anesthesiologists; Cl, confidence interval; IBD, inflammatory bowel disease.

the reported results are hardly clear and conclusive. In contrast to previous studies, we have not found any association between AL rates and the ASA score, gender, diabetes mellitus, and other comorbidities. ${ }^{4,10,29,31-33}$ The absence of this association may be explained due to an important percentage of AL having occurred in IBD patients, who most often corresponded to young patients without other comorbidities. Therefore, in elderly patients who underwent emergent TSC in obstructed or perforated colon cancer, ileostomy was preferably performed. In our study, we found patients under 50 -years-old as predictors of AL $(p=0.038)$. This may be because young IBD patients who underwent TSC might have a poorer clinical situation. Furthermore, young patients who presented emergent TSC were more eligible to primary anastomosis, with the assumption of higher risk of $\mathrm{AL}$, thus avoiding stoma creation.

No difference was identified in relation to AL when using handsewn or stapler anastomosis, which was similar to the findings of a Cochrane review and a recent meta-analysis showing no superiority of either technique. ${ }^{34,35}$ The types of stapler anastomosis (circular vs. linear) and anastomosis placement were also assessed, and no association with $\mathrm{AL}$ was found. It is still unclear if anastomosis placement might be associated with $\mathrm{AL}^{34,35}$ In our study, we found the laparoscopic approach in elective surgery as a predictor to $\mathrm{AL}(p=0.049)$. No difference has been reported between laparoscopic and open colectomies in colon cancer and IBD. ${ }^{36,37}$ Furthermore, our findings might be explained by 
Table 2 Operative data and anastomosis leakage

\begin{tabular}{|c|c|c|c|}
\hline & \multicolumn{3}{|c|}{ Anastomosis leakage } \\
\hline & $\begin{array}{l}\text { Non-anastomosis } \\
\text { leakage } \mathrm{n}(\%)\end{array}$ & $\begin{array}{l}\text { Anastomosis } \\
\text { leakage } \mathbf{n}(\%)\end{array}$ & $\begin{array}{l}p \text {-value } \\
\text { OR (CI95\%) }\end{array}$ \\
\hline Surgical approach & & & 0.16 \\
\hline Open & $56(87.5)$ & $8(12.5)$ & \\
\hline Laparoscopic & $14(73.7)$ & $5(26.3)$ & \\
\hline Anastomosis & & & 0.21 \\
\hline IRA & $25(78.1)$ & $7(21.9)$ & \\
\hline ISA & $45(88.2)$ & $6(11.8)$ & \\
\hline Anastomosis technique & & & 0.31 \\
\hline Stapler & $44(81.5)$ & $10(18.5)$ & \\
\hline Handsewn & $26(89.7)$ & $3(10.3)$ & \\
\hline Type of anastomosis & & & 0.57 \\
\hline End-to-side & $2(66.7)$ & $1(33.3)$ & \\
\hline Side-to-side & $44(83)$ & $9(17)$ & \\
\hline Side-to-end & $24(88.9)$ & $3(11.7)$ & \\
\hline Type of stapler & & & 0.89 \\
\hline Linear & $26(81.3)$ & $6(18.8)$ & \\
\hline Circular & $19(82.6)$ & $4(17.4)$ & \\
\hline \multicolumn{4}{|l|}{ Planning of operation } \\
\hline \multicolumn{4}{|l|}{ Elective } \\
\hline Open & $45(91.8)$ & $4(8.2)$ & 0,049 \\
\hline Laparoscopic & $13(72.2)$ & $5(27.8)$ & $4.32(1.01-18.49)$ \\
\hline IRA & $22(78.6)$ & $6(21.4)$ & 0.10 \\
\hline ISA & $36(92.3)$ & $3(7.7)$ & \\
\hline \multicolumn{4}{|l|}{ Emergent } \\
\hline Open & $11(73.3)$ & $4(26.7)$ & 0.44 \\
\hline Laparoscopic & $1(100)$ & 0 & \\
\hline IRA & $3(75)$ & $1(25)$ & 1.0 \\
\hline ISA & $9(75)$ & $3(25)$ & \\
\hline
\end{tabular}

Abbreviations: IRA, lleorectal anastomosis; ISA, lleosigmoid anastomosis.

the difficulty of proper placement of the small bowel over the distal stump in the laparoscopic approach. Often, the small bowel can be rotated, and it may produce insufficient perfusion or increased tension over the anastomosis. Further studies are needed to assess the potential impact factor of ISA or IRA in the laparoscopic approach on the AL rate.

In our study, we found higher AL rates in IBD patients when compared with other indications ( $p=0.009$; OR: 3.88 ). Although Nakamura et al. reported no differences in morbidity according to the underlying disease, ${ }^{12}$ some authors found a high rate of morbidity in IBD patients, from 20 to $32 \%{ }^{5,13,14,27}$ Segelman et al. used two-stage procedures in IBD patients more often than in the other indications. ${ }^{16}$ Further studies about AL risk in total or subtotal colectomies with IRA or ISA in IBD patients are needed to stratify the risk of $\mathrm{AL}$ and other postoperative complications. If a high risk of
AL is estimated, two-stage surgery (loop ileostomy) may be performed, as it has been shown to be a protective factor against $\mathrm{AL}{ }^{16}$ Further studies are necessary regarding the value of indocyanine green to prevent $A L$, improving the assessment of the rectal stump's blood supply.

Overall, the morbidity was $45.8 \%$, including medical and surgical postoperative morbidity within 90 days. No statistical differences were found in the severity of morbidity according to underlying diseases or other surgical data. Duclos et al. reported a lower morbidity rate of $19.5 \%{ }^{9}$ Even in emergency colectomy, lower rates were reported (15\%), with a high mortality rate of $9.7 \%{ }^{21}$ This heterogeneity of the reported morbidity rates shows the importance of reporting the results in detail so they can be accurately assessed. In the present report, cases of AL after IRA or ISA were often clinically severe (grade $C$ ). The same findings are 


\begin{tabular}{|c|c|c|c|c|c|c|c|c|c|c|c|c|c|c|c|c|c|c|c|c|c|c|c|c|c|c|c|c|c|c|c|c|}
\hline & 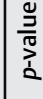 & 㐫 & & & $\underset{\sim}{\mathbb{N}}$ & & & 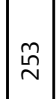 & & & & $\mid \begin{array}{l}n \\
0 \\
0 \\
0\end{array}$ & & & 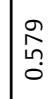 & & & $\mid \begin{array}{l} \pm \\
0 \\
0 \\
0\end{array}$ & & & $\begin{array}{l}0 \\
0 \\
0 \\
0\end{array} \mid$ & & & & & $\begin{array}{c}\hat{N} \\
\tilde{0} \\
0\end{array}$ & & 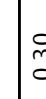 & & & 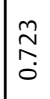 & \\
\hline \multirow{3}{*}{ 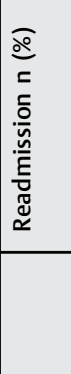 } & $\stackrel{\check{\nu}}{\nu}$ & & $\begin{array}{l}\widehat{a} \\
\dot{d} \\
\sim\end{array}$ & 0 & & 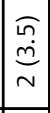 & 0 & & 0 & 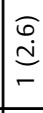 & \begin{tabular}{l}
- \\
\multicolumn{2}{c}{} \\
- \\
\end{tabular} & & 0 & $\begin{array}{l}\widehat{a} \\
\stackrel{a}{\mathrm{j}} \\
\mathrm{N}\end{array}$ & & 0 & $\begin{array}{l}0 \\
\stackrel{0}{\mathrm{j}} \\
\sim\end{array}$ & & $\begin{array}{l}\stackrel{o}{o} \\
- \\
\end{array}$ & 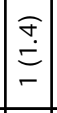 & & $\begin{array}{l}\widehat{N} \\
\stackrel{n}{\sim} \\
\sim\end{array}$ & 0 & 0 & 0 & & 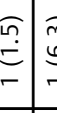 & & $\begin{array}{l}\widehat{N} \\
\stackrel{\sim}{\sim} \\
\sim\end{array}$ & 0 & & ָָ \\
\hline & i & & $\mid \begin{array}{l}2 \\
\dot{0} \\
\hat{0} \\
0 \\
0\end{array}$ & 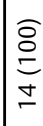 & & 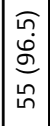 & 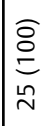 & & $\mid \begin{array}{c}\stackrel{\varrho}{\varrho} \\
\stackrel{\bar{m}}{\bar{n}}\end{array}$ & 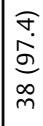 & $\begin{array}{l}\hat{\sigma} \\
\text { o } \\
\circ \\
\circ\end{array}$ & & 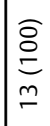 & $\begin{array}{l}\bar{\sigma} \\
\hat{\sigma} \\
6\end{array}$ & & $\begin{array}{l}\widehat{\sigma} \\
\underset{\sigma}{\sigma} \\
\sigma\end{array}$ & \begin{tabular}{l}
$\hat{\Omega}$ \\
$\hat{\alpha}$ \\
\multirow{2}{*}{}
\end{tabular} & & $\mid \begin{array}{l}\widehat{\sigma} \\
\sigma \\
\sigma\end{array}$ & $\mid \begin{array}{l}0 \\
0 \\
o \\
o \\
\kappa \\
\kappa\end{array}$ & & $\left|\begin{array}{l}0 \\
0 \\
\dot{o} \\
0 \\
0 \\
0\end{array}\right|$ & $\underset{m}{\stackrel{\Xi}{\rightleftharpoons}}$ & 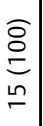 & 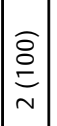 & & 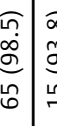 & & $\begin{array}{l}\widehat{0} \\
\dot{\leftrightarrow} \\
\bar{\sigma} \\
\bar{\sigma}\end{array}$ & $\stackrel{\Xi}{\Xi}$ & & $\begin{array}{l}\widehat{\alpha} \\
\dot{\phi}\end{array}$ \\
\hline & 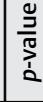 & 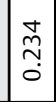 & & & $\begin{array}{c}0 \\
\hat{m} \\
\tilde{0} \\
0\end{array}$ & & & 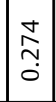 & & & & $\begin{array}{l}\hat{\sigma} \\
0 \\
0\end{array}$ & & & $\mid \begin{array}{c}0 \\
\vdots \\
0 \\
0 \\
0\end{array}$ & & & $\mid \begin{array}{l}0 \\
0 \\
0 \\
0 \\
0\end{array}$ & & & $\begin{array}{l}\text { 早 } \\
\text { م. }\end{array}$ & & & & & $\begin{array}{l}m \\
\dot{f} \\
0\end{array}$ & & 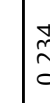 & & & $\mid$\begin{tabular}{l}
$\infty$ \\
\multirow{2}{0}{} \\
0
\end{tabular} & \\
\hline \multirow{4}{*}{ 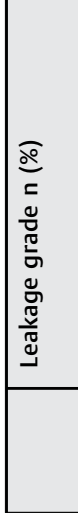 } & $u$ & & 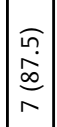 & $\frac{\widehat{D}}{\sigma}$ & & $\begin{array}{l}\pi \\
\\
6\end{array}$ & 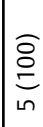 & & $\begin{array}{l}\widehat{1} \\
\infty \\
\infty \\
\infty \\
\wedge\end{array}$ & $\underset{\mathrm{m}}{\stackrel{\mathrm{o}}{\stackrel{8}{n}}}$ & 圖 & & $\underset{\sigma}{\stackrel{\widehat{o}}{c}}$ & $\mid \begin{array}{c}\tilde{m} \\
\infty \\
\infty \\
0 \\
0\end{array}$ & & $\begin{array}{l}\stackrel{0}{\circ} \\
\stackrel{0}{=} \\
\end{array}$ & 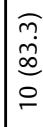 & & $\begin{array}{l}\stackrel{\varrho}{\Xi} \\
\stackrel{\Xi}{こ} \\
-\end{array}$ & 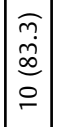 & & 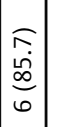 & & 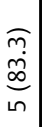 & & & & & 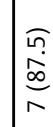 & & & \\
\hline & $\infty$ & & 0 & & & $\begin{array}{l}\mathfrak{i n} \\
\stackrel{n}{\mathfrak{I}} \\
-\end{array}$ & 0 & & 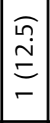 & 0 & $\circ$ & & 0 & $\mid \begin{array}{c}\tilde{m} \\
\infty \\
-\end{array}$ & & 0 & \begin{tabular}{|c}
$\widehat{\widehat{m}}$ \\
$\stackrel{\infty}{-}$
\end{tabular} & & 0 & $\mid \begin{array}{c}\widehat{m} \\
\stackrel{\infty}{\tau} \\
\end{array}$ & & 0 & & 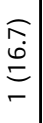 & & & 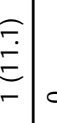 & & 0 & $\stackrel{\Xi}{\beth}$ & & $\underset{\sim}{\tilde{\sigma}}$ \\
\hline & $<$ & & 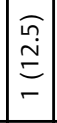 & 0 & & 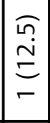 & 0 & & 0 & 0 & 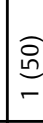 & & 0 & $\begin{array}{l}\widehat{m} \\
\infty \\
-\end{array}$ & & 0 & $\begin{array}{l}\widehat{\tilde{m}} \\
\stackrel{\infty}{-} \\
-\end{array}$ & & 0 & $\mid \begin{array}{c}\widehat{m} \\
\stackrel{\infty}{=} \\
=\end{array}$ & & 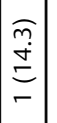 & & 0 & & & 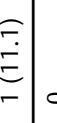 & & 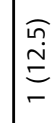 & 0 & & $\widehat{m}$ \\
\hline & 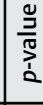 & $\begin{array}{l} \\
0 \\
0 \\
0 \\
\end{array}$ & & & 竎 & & & 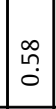 & & & & $\mid \begin{array}{c}0 \\
\tilde{0} \\
0\end{array}$ & & & $\mid \begin{array}{c}\mathbf{0} \\
\infty \\
0 \\
0 \\
0\end{array}$ & & & $\mid \begin{array}{l}\infty \\
\stackrel{0}{0} \\
0\end{array}$ & & & $\frac{\tilde{\sigma}}{\sigma}$ & & & & & 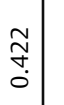 & & : & & & $\begin{array}{l}\tilde{\alpha} \\
\hat{\sigma} \\
0\end{array}$ & \\
\hline \multirow{6}{*}{$\begin{array}{l}\bar{\Xi} \\
\vdots \\
0\end{array}$} & $\geq$ & & 0 & $\stackrel{\overbrace{}}{\check{c}}$ & & $\begin{array}{l}\widehat{E} \\
\stackrel{5}{E} \\
-\end{array}$ & 0 & & $\begin{array}{c}\widehat{\tilde{n}} \\
\tilde{n} \\
- \\
\end{array}$ & 0 & $\circ$ & & 0 & \begin{tabular}{|l} 
Ð \\
ᄃ
\end{tabular} & & 0 & $\begin{array}{l}\widehat{\hat{m}} \\
\stackrel{-}{-}\end{array}$ & & 0 & $\begin{array}{c}\stackrel{\Im}{\stackrel{\Xi}{\Xi}} \\
-\end{array}$ & & 0 & 0 & $\begin{array}{l}\hat{E} \\
\dot{\varphi} \\
- \\
\end{array}$ & 0 & & 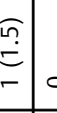 & & 0 & $\begin{array}{l}\text { m } \\
\text { ñ } \\
-\end{array}$ & & $\stackrel{\text { m. }}{=}$ \\
\hline & $\stackrel{\varrho}{=}$ & & $\mid \begin{array}{c}\widehat{\widetilde{c}} \\
\infty \\
0\end{array}$ & $\begin{array}{l}0 \\
\infty \\
\stackrel{d}{ } \\
\sigma \\
\sigma\end{array}$ & & $\begin{array}{l}0 \\
0 \\
\infty \\
\omega\end{array}$ & 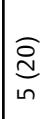 & & 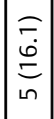 & $\underset{\sigma}{\stackrel{O}{g}}$ & - & & $\begin{array}{l}0 \\
\check{I} \\
-\end{array}$ & $\begin{array}{l}\hat{m} \\
\stackrel{\sigma}{\sigma}\end{array}$ & & $\mid \begin{array}{l}\widehat{\hat{\sigma}} \\
\underline{\omega} \\
- \\
-\end{array}$ & 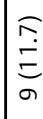 & & $\begin{array}{l}\stackrel{0}{\varrho} \\
-\end{array}$ & $\mid \begin{array}{c}\widehat{\tilde{m}} \\
\stackrel{\underline{v}}{\sigma} \\
\sigma\end{array}$ & & $\left|\begin{array}{l}\widehat{m} \\
\stackrel{\varphi}{\sigma} \\
\vec{\sigma}\end{array}\right|$ & 0 & $\begin{array}{c}\widehat{m} \\
\stackrel{m}{m} \\
\stackrel{m}{n}\end{array}$ & $\begin{array}{c}0 \\
\stackrel{a}{n} \\
-\end{array}$ & & $\begin{array}{l}\widehat{a} \\
6\end{array}$ & & $\begin{array}{l}\hat{\sigma} \\
\stackrel{\rho}{\varrho} \\
\wedge\end{array}$ & $\begin{array}{l}\infty \\
\substack{n \\
m} \\
m\end{array}$ & & กิ \\
\hline & $\leqq$ & & $\mid \begin{array}{c}\widehat{m} \\
\underset{m}{\dot{m}}\end{array}$ & 10 & & $\mid \begin{array}{l}\widehat{\widehat{c}} \\
\underline{n} \\
\mathrm{~m}\end{array}$ & 0 & & 0 & $\underset{\sim}{n}$ & $\underset{-}{-}$ & & 0 & $\underset{m}{\stackrel{\tilde{f}}{m}}$ & & 0 & $\begin{array}{l}\widehat{\widehat{m}} \\
\mathrm{~m}\end{array}$ & & 0 & $\mid \begin{array}{c}\stackrel{\mathcal{+}}{m} \\
m\end{array}$ & & 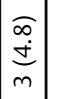 & 0 & 0 & 0 & & $\begin{array}{l}\frac{\pi}{\dot{f}} \\
m\end{array}$ & & $\underset{m}{\stackrel{a}{+}}$ & . & & 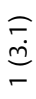 \\
\hline & $=$ & & 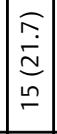 & 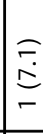 & & $\begin{array}{l}\sigma \\
\stackrel{\sigma}{\Xi} \\
=\end{array}$ & 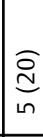 & & $\begin{array}{l}0 \\
0 \\
\dot{\Xi} \\
\end{array} \mid$ & 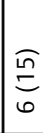 & 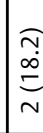 & & 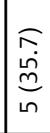 & 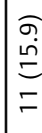 & & $\mid \begin{array}{l}\widehat{\Upsilon} \\
\dot{\omega} \\
\varrho \\
-\end{array}$ & 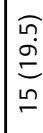 & & $\underset{\sim}{\stackrel{a}{a}}$ & $\mid \begin{array}{c}\widehat{\hat{y}} \\
\tilde{\partial} \\
\tilde{\sigma} \\
\mid\end{array}$ & & $\begin{array}{l}\partial \\
\stackrel{\Xi}{\simeq} \\
\simeq\end{array}$ & $\begin{array}{l}\widehat{m} \\
\stackrel{m}{n} \\
- \\
-\end{array}$ & $\underset{m}{\stackrel{\Xi}{a}}$ & 0 & & 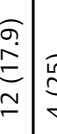 & & 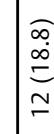 & $\underset{\bar{d}}{\bar{d}}$ & & $\begin{array}{l}\widehat{\infty} \\
\infty \\
\infty\end{array}$ \\
\hline & - & & 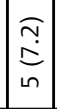 & $\underset{c}{\stackrel{\overbrace{}}{\check{c}}}$ & & $\begin{array}{l}\sigma \\
\dot{\sigma} \\
\dot{\varphi} \\
\sigma\end{array}$ & $\stackrel{\infty}{\sim}$ & & $\mid \begin{array}{l}\tilde{\Lambda} \\
\dot{\varphi} \\
\sim\end{array}$ & $\underset{\sigma}{\stackrel{\rho}{\rho}}$ & 0 & & 0 & $\mid \begin{array}{l}\hat{R} \\
\infty \\
6\end{array}$ & & 0 & $\begin{array}{l}\widehat{\infty} \\
\stackrel{\infty}{\sigma} \\
0\end{array}$ & & 0 & $\mid \begin{array}{c}\widehat{x} \\
0 \\
0 \\
0\end{array}$ & & 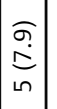 & $\begin{array}{c}\widehat{m} \\
\underset{m}{m} \\
- \\
-\end{array}$ & 0 & 0 & & 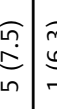 & & 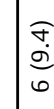 & 0 & & $\begin{array}{l}\widehat{F} \\
\stackrel{\sigma}{\sigma} \\
\sigma\end{array}$ \\
\hline & 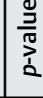 & 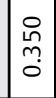 & & & $\begin{array}{l}\Re \\
\hat{0}\end{array}$ & & & $\mid \begin{array}{l}\overline{\underline{\sigma}} \\
0 \\
\dot{0}\end{array}$ & & & & $\mid \begin{array}{l}\infty \\
\stackrel{N}{N} \\
0\end{array}$ & & & 贻 & & & \begin{tabular}{|l|}
$\mid \begin{array}{c}\mid \\
0 \\
0 \\
0\end{array}$ \\
\end{tabular} & & & $\begin{array}{l}0 \\
\vdots \\
\vdots \\
0 \\
0\end{array}$ & & & & & 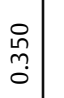 & & ה & & & 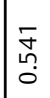 & \\
\hline \multirow{3}{*}{ 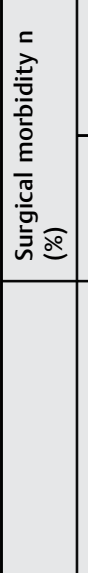 } & $\stackrel{\Delta}{\varnothing}$ & & $\begin{array}{c}\widehat{n} \\
\tilde{y} \\
\tilde{y} \\
0 \\
0\end{array}$ & $\begin{array}{l}\tilde{i} \\
\stackrel{n}{n} \\
\infty\end{array}$ & & $\begin{array}{l}\widehat{\infty} \\
\dot{J} \\
\dot{J} \\
\stackrel{0}{v} \\
\end{array}$ & $\begin{array}{l}\widehat{\infty} \\
\infty \\
o \\
\stackrel{d}{d} \\
\simeq \\
\simeq\end{array}$ & & $\mid \begin{array}{c}0 \\
0 \\
\vdots \\
0 \\
0 \\
-\end{array}$ & 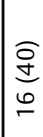 & 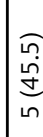 & & $\mid \begin{array}{l}\stackrel{a}{\circ} \\
\stackrel{n}{n}\end{array}$ & 命 & & $\begin{array}{l}\widehat{m} \\
\tilde{m} \\
\tilde{n} \\
\sim\end{array}$ & 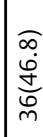 & & 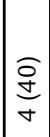 & $\mid \begin{array}{l}0 \\
\dot{0} \\
0 \\
\dot{0} \\
\dot{m} \\
m\end{array}$ & & 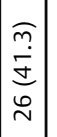 & $\begin{array}{l}\mathbb{1} \\
\dot{b} \\
\dot{b} \\
\sim\end{array}$ & $\begin{array}{l}\widehat{\widehat{o}} \\
\vec{\sigma}\end{array}$ & $\mid \begin{array}{c}0 \\
\stackrel{0}{0} \\
-\end{array}$ & & 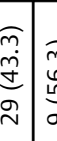 & & $\begin{array}{l}\hat{a} \\
\dot{b} \\
0 \\
\dot{m}\end{array}$ & 亲 & & 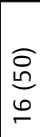 \\
\hline & i & & 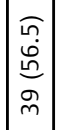 & 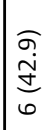 & & 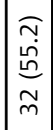 & $\begin{array}{l}\widehat{N} \\
\tilde{N} \\
\underline{n} \\
m\end{array}$ & & 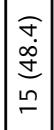 & 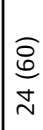 & 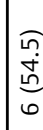 & & $\mid \begin{array}{l}\widehat{O} \\
\\
r\end{array}$ & 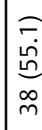 & & $\mid \begin{array}{l}\hat{T} \\
\dot{\theta} \\
\sigma \\
\sigma\end{array}$ & $\begin{array}{l}\widehat{\tilde{N}} \\
\tilde{n} \\
\bar{\sigma}\end{array}$ & & $\mid \begin{array}{l}0 \\
0 \\
0 \\
0\end{array}$ & 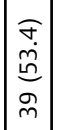 & & $\begin{array}{l}\widehat{r} \\
\infty \\
\infty \\
\hat{n} \\
m\end{array}$ & $\begin{array}{c}\widehat{m} \\
\tilde{n} \\
- \\
-\end{array}$ & 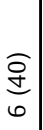 & $\begin{array}{c}\widehat{o} \\
\stackrel{0}{n} \\
-\end{array}$ & & 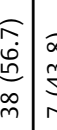 & & 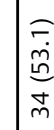 & $\begin{array}{l}\sigma \\
\hat{n} \\
=\end{array}$ & & - \\
\hline & & 茕 & 吕 & in & 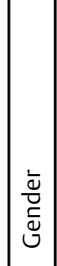 & $\frac{0}{\frac{\pi}{\pi}}$ & \begin{tabular}{|l|}
$\frac{0}{\widetilde{N}}$ \\
$\frac{\tilde{E}}{4}$
\end{tabular} & 离 & - & $=$ & $\equiv$ & 号 & $\stackrel{\tilde{y}}{x}$ & $\stackrel{2}{2}$ & 站 & $\stackrel{\check{\nu}}{\nu}$ & 2 & 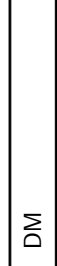 & $\stackrel{\tilde{\Perp}}{\nu}$ & 2 & 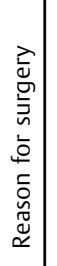 & 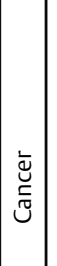 & 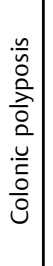 & $\stackrel{\varrho}{\varrho}$ & 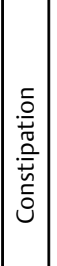 & 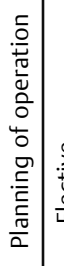 & 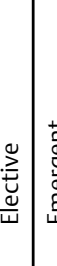 & 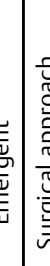 & 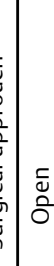 & 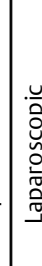 & 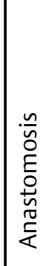 & 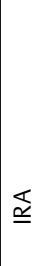 \\
\hline
\end{tabular}


44 Risk Factors for Anastomotic Leakage Ocaña et al.

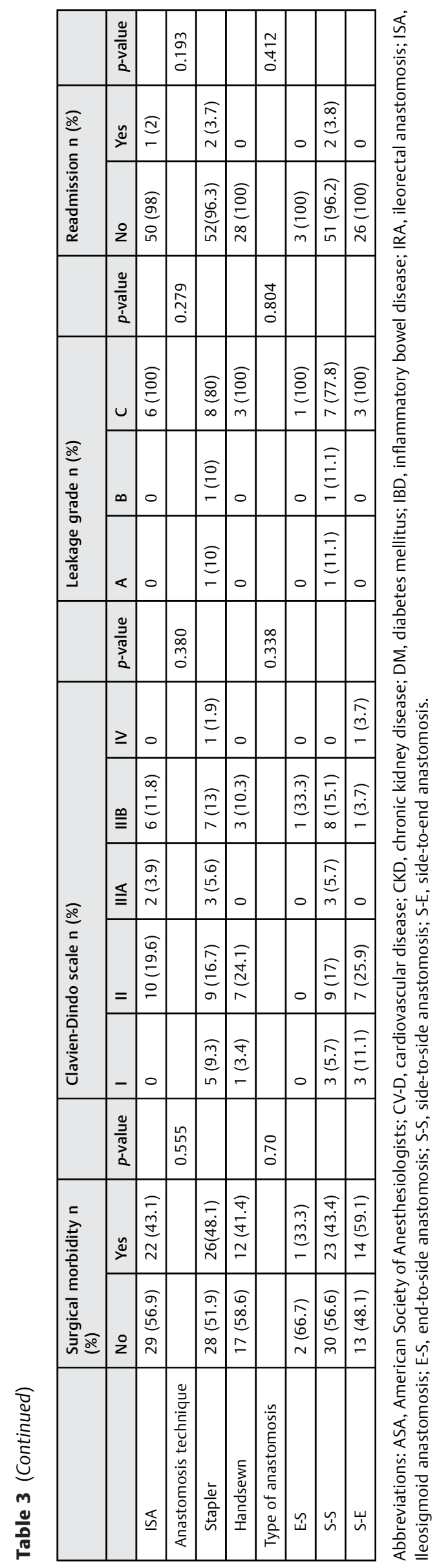


Table 4 Postoperative morbidity and complications

\begin{tabular}{|l|l|}
\hline Variables & N (\%) \\
\hline Overall morbidity & $38(45.8)$ \\
\hline Anastomotic leakage & $13(15.6)$ \\
\hline Grade A & $3(3.6)$ \\
\hline Grade B & $2(2.4)$ \\
\hline Grade C & $11(13.2)$ \\
\hline Management of anastomotic leakage & \\
\hline Surgical & $11 / 13(13.2)$ \\
\hline End ileostomy & $7(8.4)$ \\
\hline Drainage & $1(1.2)$ \\
\hline Diverting ileostomy & $3(3.6)$ \\
\hline Urinary retention & $1(1.2)$ \\
\hline Abdominal wall hematoma & $1(1.2)$ \\
\hline Intra-abdominal abscess & $1(1.2)$ \\
\hline Wound infection & $4(4.82)$ \\
\hline Intra-abdominal bleeding & $1(1.2)$ \\
\hline Lower intestinal bleeding & $1(1.2)$ \\
\hline Gastroesophageal reflux & $1(1.2)$ \\
\hline Supraventricular tachycardia & $1(1.2)$ \\
\hline Wound herniation & $2(2.4)$ \\
\hline
\end{tabular}

reported after TSC by Duclos et al., $6.8 \%$ from a morbidity rate of $19.5 \%,{ }^{9}$ and by Bakker et al., who reported a mortality rate of $13 \%$ for AL following TSC. ${ }^{4}$

The strength of the present study is that it is one of the largest series published in last years focused on restorative TSC. Even though a great effort has been made to correct possible bias in the study design, its retrospective nature will have introduced an element of bias. A large cohort, including various types of indications for surgery, might cause confusion, but it can help assess the impact of the pathology on the results. Possible confounders, such as two-stage procedures or delayed ISA or IRA, have been excluded from the study. The deep assess of the surgical morbidity may not have enough power in this sample. For this reason, large multicenter prospective studies should be made to determine pathology and surgical data impact on the type of morbidity. The applied definition criteria for $\mathrm{AL}$ may underdiagnose $\mathrm{AL}$, as not all suspicions of AL were identified by a radiological examination with rectal contrast.

The global AL rate of $15.6 \%$ after TSC in this study shows a high frequency of leakage after a relatively safe surgical procedure. Our findings suggest that, in IBD patients, the risk of AL may be higher. According to our results, identifying risk factors prior to surgery may improve short-term negative outcomes.

\section{Funding}

No funding or grant was received for the preparation of this manuscript.

\section{Conflict of Interests}

The authors have no conflict of interests to declare.

\section{References}

1 Raahave D, Loud FB, Christensen E, Knudsen LL. Colectomy for refractory constipation. Scand J Gastroenterol 2010;45(05): 592-602

2 Moszkowicz D, Mariani A, Trésallet C, Menegaux F. Ischemic colitis: the ABCs of diagnosis and surgical management. J Visc Surg 2013;150(01):19-28

3 Washington C, Carmichael JC. Management of ischemic colitis. Clin Colon Rectal Surg 2012;25(04):228-235

4 Bakker IS, Grossmann I, Henneman D, Havenga K, Wiggers T. Risk factors for anastomotic leakage and leak-related mortality after colonic cancer surgery in a nationwide audit. Br J Surg 2014;101 (04):424-432, discussion 432

5 Elton C, Makin G, Hitos K, Cohen CRG. Mortality, morbidity and functional outcome after ileorectal anastomosis. Br J Surg 2003; 90(01):59-65

6 Mirnezami A, Mirnezami R, Chandrakumaran K, Sasapu K, Sagar P, Finan P. Increased local recurrence and reduced survival from colorectal cancer following anastomotic leak: systematic review and meta-analysis. Ann Surg 2011;253(05):890-899

7 Law WL, Choi HK, Lee YM, Ho JWC, Seto CL. Anastomotic leakage is associated with poor long-term outcome in patients after curative colorectal resection for malignancy. J Gastrointest Surg 2007;11 (01):8-15

8 Lu ZR, Rajendran N, Lynch AC, Heriot AG, Warrier SK. Anastomotic leaks after restorative resections for rectal cancer compromise cancer outcomes and survival. Dis Colon Rectum 2016;59(03): 236-244

9 Duclos J, Lefevre JH, Lefrançois M, et al. Immediate outcome, longterm function and quality of life after extended colectomy with ileorectal or ileosigmoid anastomosis. Colorectal Dis 2014;16 (08):0288-0296

102015 European Society of Coloproctology Collaborating Group. Predictors for Anastomotic Leak, Postoperative Complications, and Mortality After Right Colectomy for Cancer: Results From an International Snapshot Audit. Dis Colon Rectum 2020;63(05): 606-618

11 Platell C, Barwood N, Dorfmann G, Makin G. The incidence of anastomotic leaks in patients undergoing colorectal surgery. Colorectal Dis 2007;9(01):71-79

12 Nakamura T. Are complications of TSC with ileorectal anastomosis related to the original disease? Am Surg 2001

13 Pastore RLO, Wolff BG, Hodge D. Total abdominal colectomy and ileorectal anastomosis for inflammatory bowel disease. Dis Colon Rectum 1997;40(12):1455-1464

14 Loftus EV Jr, Delgado DJ, Friedman HS, Sandborn WJ. Colectomy and the incidence of postsurgical complications among ulcerative colitis patients with private health insurance in the United States. Am J Gastroenterol 2008;103(07):1737-1745

15 Björk J, Åkerbrant $\mathrm{H}$, Iselius L, et al. Outcome of primary and secondary ileal pouch-anal anastomosis and ileorectal anastomosis in patients with familial adenomatous polyposis. Dis Colon Rectum 2001;44(07):984-992

16 Segelman J, Mattsson I, Jung B, Nilsson PJ, Palmer G, Buchli C. Risk factors for anastomotic leakage following ileosigmoid or ileorectal anastomosis. Colorectal Dis 2018;20(04):304-311

17 Rahbari NN, Weitz J, Hohenberger W, et al. Definition and grading of anastomotic leakage following anterior resection of the rectum: a proposal by the International Study Group of Rectal Cancer. Surgery 2010;147(03):339-351

18 Kulu Y, Ulrich A, Bruckner T, et al; International Study Group of Rectal Cancer. Validation of the International Study Group of Rectal Cancer definition and severity grading of anastomotic leakage. Surgery 2013;153(06):753-761 
19 Dindo D, Demartines N, Clavien PA. Classification of surgical complications: a new proposal with evaluation in a cohort of 6336 patients and results of a survey. Ann Surg 2004;240(02): 205-213

20 von Elm E, Altman DG, Egger M, Pocock SJ, Gøtzsche PC, Vandenbroucke JPSTROBE Initiative. The strengthening the reporting of observational studies in epidemiology (STROBE) statement: Guidelines for reporting observational studies. Int J Surg 2014; 12(12):1495-1499

21 Hennekinne-Mucci S, Tuech JJ, Bréhant O, et al. Emergency subtotal/total colectomy in the management of obstructed left colon carcinoma. Int J Colorectal Dis 2006;21(06):538-541

22 O'Riordan JM, O'Connor BI, Huang H, et al. Long-term outcome of colectomy and ileorectal anastomosis for Crohn's colitis. Dis Colon Rectum 2011;54(11):1347-1354

23 Boccola MA, Buettner PG, Rozen WM, et al. Risk factors and outcomes for anastomotic leakage in colorectal surgery: a single-institution analysis of 1576 patients. World J Surg 2011;35 (01):186-195

24 Torralba JA, Robles R, Parrilla P, et al. TSC vs. intraoperative colonic irrigation in the management of obstructed left colon carcinoma. Dis Colon Rectum 1998

25 Stephenson BM, Shandall AA, Farouk R, Griffith G. Malignant leftsided large bowel obstruction managed by subtotal/total colectomy. Br J Surg 1990;77(10):1098-1102

26 Ross S, Krukowski ZH, Munro A, Russell IT. Single-stage treatment for malignant left-sided colonic obstruction: A prospective randomized clinical trial comparing TSC with segmental resection following intraoperative irrigation. Br J Surg 1995

27 Yamamoto T, Keighley MRB. Proctocolectomy is associated with a higher complication rate but carries a lower recurrence rate than total colectomy and ileorectal anastomosis in Crohn colitis. Scand J Gastroenterol 1999;34(12):1212-1215
28 Krarup PM, Nordholm-Carstensen A, Jorgensen LN, Harling H. Association of comorbidity with anastomotic leak, 30-day mortality, and length of stay in elective surgery for colonic cancer: A nationwide cohort study. Dis Colon Rectum 2015;58(07):668-676

29 Sparreboom CL, van Groningen JT, Lingsma HF, et al; Dutch ColoRectal Audit group. Different risk factors for early and late colorectal anastomotic leakage in a nationwide audit. Dis Colon Rectum 2018;61(11):1258-1266

30 Sciuto A, Merola G, De Palma GD, et al. Predictive factors for anastomotic leakage after laparoscopic colorectal surgery. World J Gastroenterol 2018;24(21):2247-2260

31 Trencheva K, Morrissey KP, Wells M, et al. Identifying important predictors for anastomotic leak after colon and rectal resection: prospective study on 616 patients. Ann Surg 2013;257(01):108-113

32 Lin X, Li J, Chen W, et al. Diabetes and risk of anastomotic leakage after gastrointestinal surgery. J Surg Res 2015;196(02):294-301

33 McDermott FD, Heeney A, Kelly ME, Steele RJ, Carlson GL, Winter DC. Systematic review of preoperative, intraoperative and postoperative risk factors for colorectal anastomotic leaks. Br J Surg 2015;102(05):462-479

34 Neutzling CB, Lustosa SA, Proenca IM, da Silva EM, Matos D. Stapled versus handsewn methods for colorectal anastomosis surgery. Cochrane Database Syst Rev 2012;(02):CD003144

35 Naumann DN, Bhangu A, Kelly M, Bowley DM. Stapled versus handsewn intestinal anastomosis in emergency laparotomy: a systemic review and meta-analysis. Surgery 2015;157(04):609-618

36 Watanabe K, Funayama Y, Fukushima K, Shibata C, Takahashi KI, Sasaki I. Hand-assisted laparoscopic vs. open TSC for severe ulcerative colitis. Dis Colon Rectum 2009

37 Veldkamp R, Kuhry E, Hop WC, et al; COlon cancer Laparoscopic or Open Resection Study Group (COLOR) Laparoscopic surgery versus open surgery for colon cancer: short-term outcomes of a randomised trial. Lancet Oncol 2005;6(07):477-484 